\title{
Hepatoid adenocarcinoma of the stomach
}

\author{
Satoshi Inagawa ${ }^{1,3}$, Jiro Shimazaki ${ }^{1}$, Masao Hori ${ }^{1}$, Fuyo Yoshimi ${ }^{2}$, Shinya Adachi ${ }^{3}$, Toru Kawamoto ${ }^{3}$, \\ Katashi FuKaO ${ }^{3}$, and Masayuki ItABASHi ${ }^{1}$ \\ ${ }^{1}$ Department of Pathology, Ibaraki Prefectural Central Hospital and Cancer Center, 6528 Koibuchi, Tomobe-machi, Ibaraki 309-1793, Japan \\ ${ }^{2}$ Department of Surgery, Ibaraki Prefectural Central Hospital and Cancer Center, Ibaraki, Japan \\ ${ }^{3}$ Department of Surgery, Institute of Clinical Medicine, University of Tsukuba, Ibaraki, Japan
}

\begin{abstract}
Although gastric cancer occurs frequently in Japan, few cases of hepatoid adenocarcinoma, a cancer with an extremely poor prognosis, have been reported. Here, we describe a 67-yearold Japanese man referred to our hospital with suspected gastric cancer. Gastrointestinal fiberscopy revealed an elevated lesion with a central depression on the lesser curvature, extending from the antrum to the body of the stomach. On the preoperative examinations, abdominal computed tomography scan, magnetic resonance imaging, and abdominal ultrasonography revealed multiple metastases to the liver and no cirrhotic change. The serum level of alpha-fetoprotein (AFP) was markedly elevated $(10084 \mathrm{ng} / \mathrm{ml})$. After a diagnosis of AFP-producing gastric cancer with multiple liver metastases was made, total gastrectomy, without liver resection, was performed. Microscopically, the tumor showed two main histological features. The main part of the tumor resembled moderately differentiated hepatocellular carcinoma, and the rest showed fetal-type adenocarcinoma. Some parts of the hepatoma-like lesion showed periodic acid-Schiff (PAS)positive granules. Furthermore, the tumor showed diffuse immunohistochemical positivity for AFP, alpha-1 antitrypsin, and alpha-1 antichymotrypsin. According to these histopathological findings, the tumor was diagnosed as hepatoid adenocarcinoma of the stomach. Although anastomotic leakage occurred postoperatively and the liver metastases have increased in size, the patient remains alive 11 months after the operation. Because of the poor prognosis for this histological type of tumor, accurate diagnosis of hepatoid adenocarcinoma is important, and long-term follow-up is required. We describe this rare case of hepatoid adenocarcinoma of the stomach, and review the literature concerning the clinicopathological aspects.
\end{abstract}

Key words Gastric cancer - Hepatoid adenocarcinoma • Alpha-fetoprotein-producing cancer $\cdot$ Stomach

Offprint requests to: S. Inagawa, Department of Surgery, Tsukuba Medical Center Hospital, 1-3-1 Amakubo, Tsukuba, Ibaraki 305-8558, Japan

Received: January 9, 2001 / Accepted: March 13, 2001

\section{Introduction}

Alpha-fetoprotein (AFP) is a fetal serum protein produced by fetal liver and yolk sac cells, and by some fetal gastrointestinal cells [1]. After birth, the level of the protein in serum rapidly decreases. However, its level is elevated in patients with hepatocellular carcinoma and in those with noncancerous liver diseases associated with liver regeneration, such as cirrhosis and hepatitis [2-6]. Recently, AFP-producing tumors have been reported in several different organs [7-13], with gastric adenocarcinoma being one of the most common of these tumors [14]. Since the first report of AFPproducing gastric carcinoma by Bourreille et al. [15], other cases have been described, and the incidence is reported to be $1.3 \%-15 \%$ of all gastric carcinomas [1620]. Kodama et al. [21] described two histologic types of AFP-producing gastric carcinoma. Later, Ishikura et al. $[22,23]$ proposed the term "hepatoid adenocarcinoma of the stomach" for primary gastric carcinomas that are characterized by both hepatoid differentiation and the production of large amounts of AFP. This type of tumor is a relatively rare gastric carcinoma, and is recognized as having a poor prognosis, even if the tumor is diagnosed at an early stage [24]. In this report, we describe a rare case of hepatoid adenocarcinoma of the stomach, with a review of the literature and investigation of the clinical features.

\section{Case report}

A 67-year-old Japanese man consulted a local doctor because of a 2-month history of general fatigue. Upper gastrointestinal radiological studies suggested gastric cancer (Fig. 1A). He was referred to our hospital on February 9, 2000, for further examination. His medical history included hypertension and diabetes. 
On physical examination, anemic conjunctiva was detected. Results of the physical examinations were unremarkable. Hematological investigations revealed anemia (hemoglobin $[\mathrm{Hb}], 7.6 \mathrm{~g} / \mathrm{dl}$; hematocrit [Ht], $26.2 \%)$. Results of other laboratory tests, including liver function tests, were all within normal limits. The serum level of AFP was elevated $(10084 \mathrm{ng} / \mathrm{ml})$, but the levels of carbohydrate antigen 19-9 (CA19-9) and carcinoembryonic antigen (CEA) were within normal limits. Hepatitis B surface antigen and antibody, and hepatitis $\mathrm{C}$ antibody, were all negative. Endoscopy (Fig. 1B) revealed an elevated tumor, with foci of necrosis and hemorrhage, at the lesser curvature, extending from the antrum to the body of the stomach. The tumor was classified macroscopically as type 5 , according to the general rules of the Japanese Gastric Cancer Association (JGCA) [25]. Gastric biopsy revealed moderatelyto-poorly differentiated adenocarcinoma. Abdominal computed tomography (CT) scan, ultrasonography, and magnetic resonance imaging (MRI) showed thickening of the posterior wall of the stomach, lymph node swelling around the left gastric artery and common hepatic artery, and a $7.0-\mathrm{cm}$ cyst in the upper pole of the right kidney. Tumor infiltration to the pancreas was not evident. Multiple solid lesions, highly suggestive of metastasis from gastric cancer, were revealed in the liver (Fig. 2), but no cirrhotic change was observed. A diagnosis of AFP-producing gastric cancer with multiple liver metastases was made, and total gastrectomy with lymph node dissection was performed, on February 18, 2000.
At the operation, neither fibrotic change nor cirrhosis was seen in the liver. Because of the multiple metastases, liver resection was not performed. The surgically resected specimen showed an elevated tumor, $8.5 \mathrm{~cm}$ in maximal diameter, with central ulceration and surface erosion, involving the antrum and body, from the lesser curvature to the posterior-wall. There was a polypoid tumorous component in the posterior-wall side of the lesion (Fig. 3A). The tumor was classified as type 5 , according to the general rules of the JGCA.

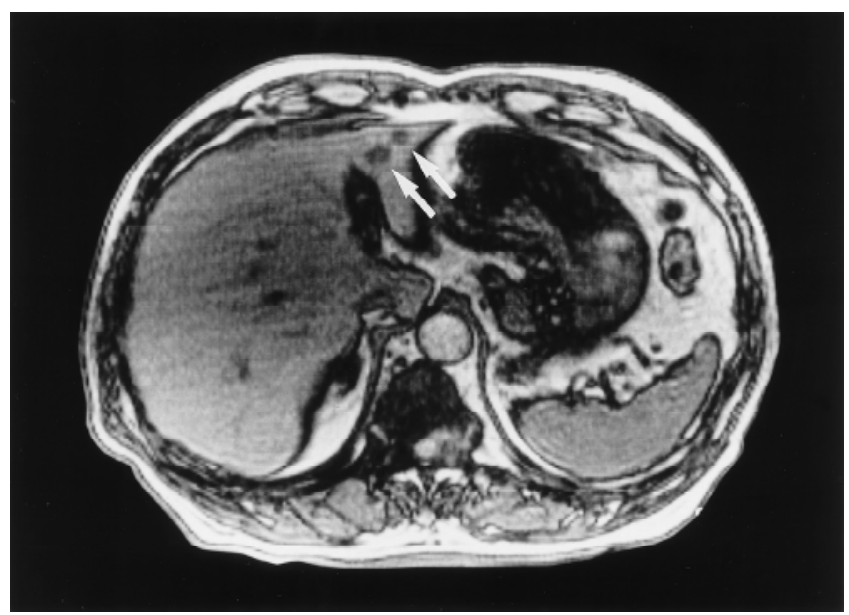

Fig. 2. Magnetic resonance imaging (MRI) scan of the liver, showing multiple solid low-intensity lesions in the left lobe of the liver (arrows)
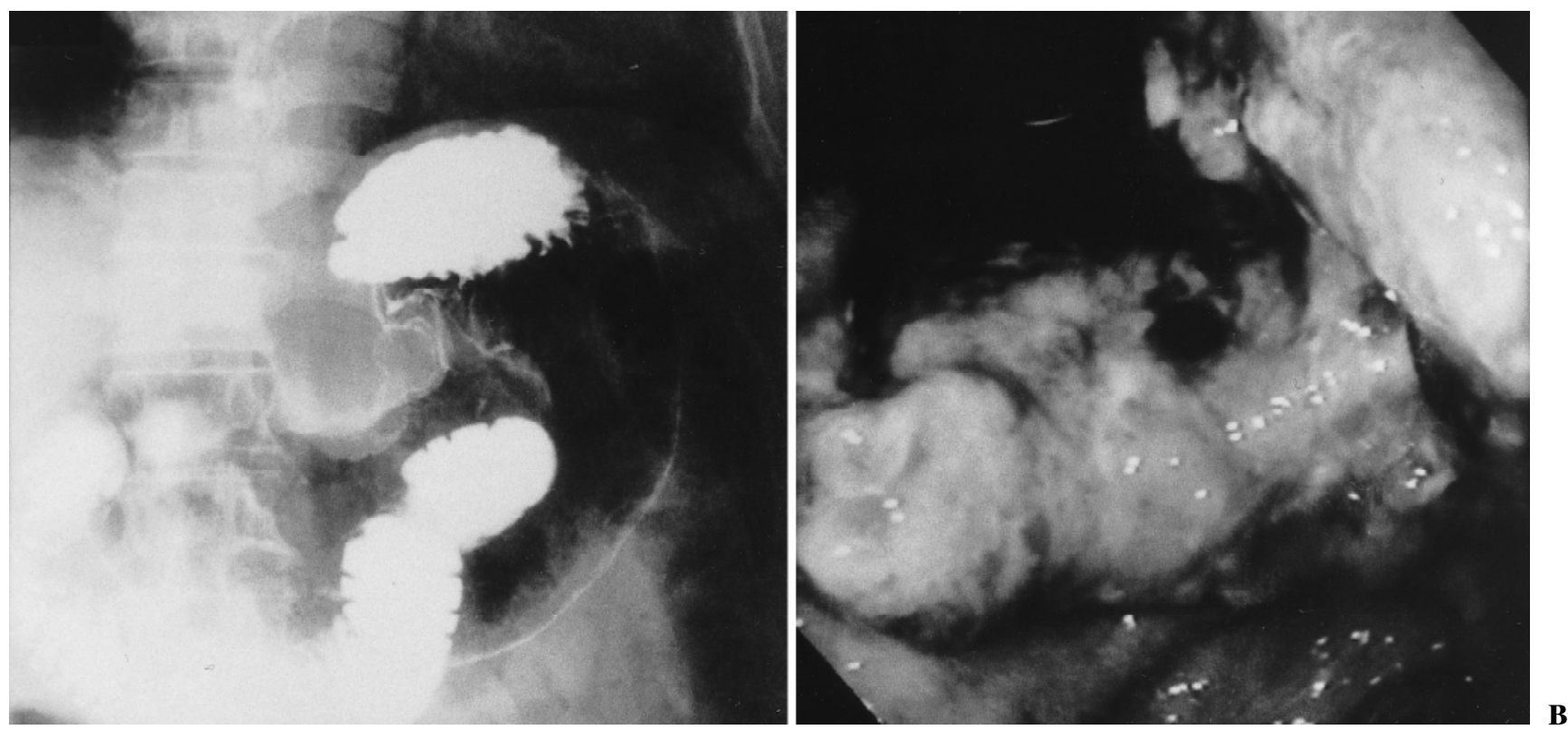

Fig. 1. A Upper gastrointestinal radiological study, showing an elevated tumor at the lesser curvature, extending from the antrum to the body of the stomach. B Gastroduodenal fiberscopy reveals an elevated tumor, with central depression and erosion, at the lesser curvature of the antrum 
$\mathbf{A}$
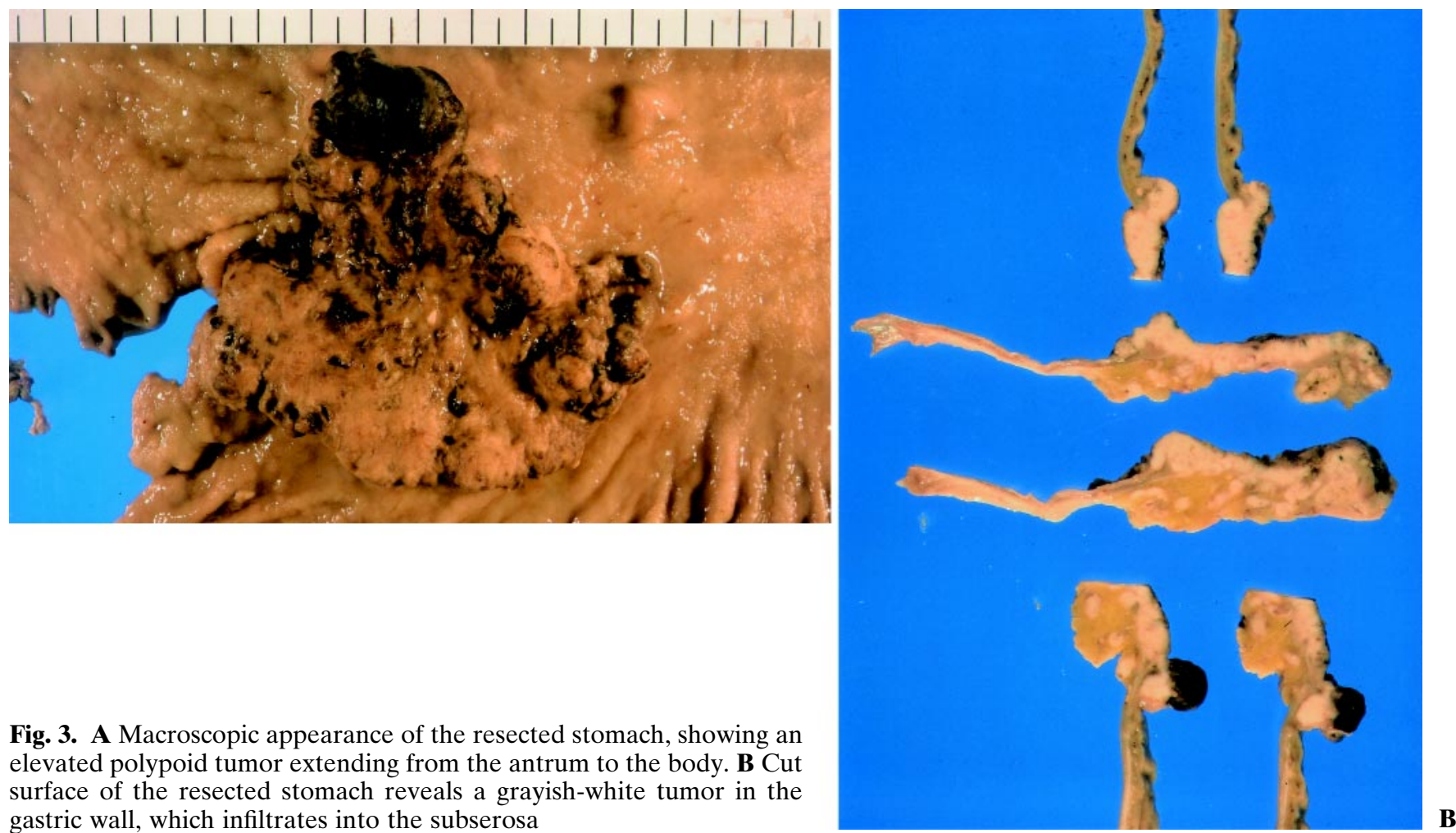

Fig. 3. A Macroscopic appearance of the resected stomach, showing an elevated polypoid tumor extending from the antrum to the body. B Cut surface of the resected stomach reveals a grayish-white tumor in the gastric wall, which infiltrates into the subserosa

The cut surface showed a slightly grayish-white tumor infiltrating to at least the muscularis propria (Fig. 3B). Areas of coagulation necrosis were seen in the tumor. Grossly, the remainder of the resected stomach appeared unremarkable. Three lymph nodes on the lesser curvature and one lymph node on the greater curvature showed massive swelling.

Light microscopic examination showed that tumor invasion was mainly limited to the area from the mucosa to the proper muscle layer, although invasion to the subserosal layer was evident in some areas. The tumor showed three types of histology. The major part, composed of large polygonal cells with abundant eosinophilic cytoplasm separated by fibrous stroma, arranged in a trabecular pattern, resembled moderately differentiated hepatocellular carcinoma (Fig. 4A). One of the other parts showed moderately differentiated fetal-type adenocarcinoma with clear cell foci, and also showed large-cell transformation (Fig. 4B), while an area of common-type adenocarcinoma was seen in less than $10 \%$ of the whole tumor. Coagulation necrosis was seen in many areas of the tumor nodules. Prominent intravascular proliferation of hepatoid tumor cells, which sometimes formed tumor thrombi, was frequently observed. Neither a reticular pattern nor Schiller-Duval bodies were found in the tumor. Occasional cells in both the hepatoid and fetal-type components had intracyto- plasmic hyaline globules, which were stained with PAS and resistant to diastase digestion. Despite further detailed study by light microscopy, bile secretion was not observed. Immunohistochemistry showed that both the hepatocellular carcinoma-like cells and fetal-type adenocarcinoma cells were diffusely positive for AFP (Fig. 5). Furthermore, alpha-1 antitrypsin (AAT) and alpha1 antichymotripsin (ACT) were focally positive in the hepatoid areas. Specific staining for CEA was focally demonstrated in the peripheral adenocarcinomatous area. Ki-67 expression in the tumor cell nuclei was markedly elevated (Ki-67 labeling index, 60.2\%). On the basis of these pathologic and immunohistochemical findings, the tumor was diagnosed as hepatoid adenocarcinoma of the stomach. There were cancer metastases in 4 of the 73 lymph nodes histologically examined from the lesser curvature and near the common hepatic artery. The tumor was diagnosed as stage IV (ss, ly2, v3, n2, H1, M0) according to the general rules of the JGCA [25].

Postoperatively, anastomotic leakage occurred at the esophagojejunostomy site, but it was controlled by the injection of fibrin adhesive into the area surrounding the anastomosis. The patient was then treated orally with an anticancer drug (1 M tegafur- $0.4 \mathrm{M}$ gimestat$1 \mathrm{M}$ ostat potassium, TS-1). Although the serum AFP level decreased to $6.2 \mathrm{ng} / \mathrm{ml}$ within 2 months, it has 
Table 1. Summary of patients with hepatoid adenocarcinoma of the stomach

\begin{tabular}{|c|c|c|c|c|c|c|c|c|c|}
\hline \multirow[b]{2}{*}{ Author } & \multirow[b]{2}{*}{ Year } & \multirow[b]{2}{*}{$\begin{array}{l}\text { Number of } \\
\text { patients }\end{array}$} & \multirow[b]{2}{*}{$\begin{array}{l}\text { Age } \\
\text { (years) }\end{array}$} & \multirow[b]{2}{*}{ Sex } & \multirow[b]{2}{*}{ Chief complaints } & \multirow[b]{2}{*}{ Location } & \multicolumn{3}{|c|}{ Tumor marker } \\
\hline & & & & & & & $\begin{array}{c}\text { AFP } \\
(\mathrm{ng} / \mathrm{ml})\end{array}$ & $\begin{array}{l}\text { PIVKA-II } \\
\text { (U/ml) }\end{array}$ & $\begin{array}{c}\text { CEA } \\
(\mathrm{ng} / \mathrm{ml})\end{array}$ \\
\hline Ishikura et al. [22] & 1985 & 1 & 63 & M & Fatigue & $\mathrm{L}$ & 27000 & NA & 5 \\
\hline \multirow[t]{6}{*}{ Ishikura et al. [23] } & 1986 & 6 & 67 & M & NA & $\mathrm{U}$ & 4730 & NA & 0.2 \\
\hline & & & 48 & M & NA & Whole & 6400 & NA & NA \\
\hline & & & 59 & $\mathrm{~F}$ & NA & $\mathrm{L}$ & 700000 & NA & NA \\
\hline & & & 77 & $\mathrm{~F}$ & NA & $\mathrm{L}$ & 10600 & NA & 3.6 \\
\hline & & & 44 & $\mathrm{~F}$ & NA & $\mathrm{L}$ & 12000 & NA & NA \\
\hline & & & 68 & M & NA & $\mathrm{L}$ & 10000 & NA & 10.0 \\
\hline Ebihara et al. [30] & 1987 & 1 & 70 & $\mathrm{M}$ & Nausea & $\mathrm{M}$ & 2888 & NA & 1.4 \\
\hline \multirow[t]{2}{*}{ Ishikura et al. [31] } & 1987 & 2 & 48 & $\mathrm{~F}$ & NA & $\mathrm{U}$ & 2780 & NA & 1.5 \\
\hline & & & 65 & $\mathrm{~F}$ & NA & $\mathrm{L}$ & 8000 & NA & NA \\
\hline Yamada et al. [32] & 1987 & 1 & 72 & M & Fatigue & $\mathrm{L}$ & 553888 & NA & 9.1 \\
\hline Watanabe et al. [33] & 1988 & 1 & 63 & M & Epigastric pain & LM & 12240 & NA & 0.3 \\
\hline Ohashi et al. [34] & 1988 & 1 & 76 & M & Fatigue & $\mathrm{L}$ & 1896.9 & NA & 1.0 \\
\hline Yamagiwa [35] & 1988 & 1 & 55 & M & Edema & $\mathrm{U}^{1}$ & 6.18 & NA & 10.4 \\
\hline $\mathrm{Li}[36]$ & 1989 & 1 & 57 & M & $\begin{array}{l}\text { Abdominal mass, } \\
\text { anorexia }\end{array}$ & $\mathrm{L}$ & 652.8 & NA & NA \\
\hline Matias-Guiu et al. [37] & 1989 & 1 & 54 & $\mathrm{~F}$ & $\begin{array}{l}\text { Gastrointestinal } \\
\text { bleeding }\end{array}$ & $\mathrm{L}$ & NA & NA & NA \\
\hline Takeuchi et al. [38] & 1989 & 1 & 63 & M & Fatigue & $\mathrm{U}$ & 37000 & NA & 1.0 \\
\hline Miyake et al. [39] & 1989 & 1 & 71 & M & Epigastric pain & ML & 35000 & NA & Normal \\
\hline Caruso $[40]$ & 1991 & 1 & 75 & $\mathrm{~F}$ & $\begin{array}{l}\text { Epigastric pain, } \\
\text { BW loss }\end{array}$ & $\mathrm{L}$ & NA & NA & NA \\
\hline \multirow{3}{*}{ Chang et al. [24] } & 1991 & 3 & 62 & $\mathrm{~F}$ & Epigastric pain & M & 146 & NA & NA \\
\hline & & & 59 & M & AFP increase & $\mathrm{L}$ & 4800 & NA & NA \\
\hline & & & 65 & M & Epigastric pain & $\mathrm{L}$ & $<1$ & NA & NA \\
\hline Votte et al. [41] & 1991 & 1 & 67 & NA & NA & NA & 13245 & NA & NA \\
\hline Kudo et al. [42] & 1992 & 1 & 56 & M & Back pain & $\mathrm{L}$ & 2810 & 2.45 & 3.2 \\
\hline de Lorimier et al. [43] & 1993 & 1 & 54 & M & $\begin{array}{l}\text { Abdominal pain, } \\
\text { BW loss }\end{array}$ & Whole & NA & NA & NA \\
\hline \multirow{8}{*}{ Motoyama et al. [26] } & 1993 & 1 & 71 & $\mathrm{~F}$ & Abdominal mass & $\mathrm{L}$ & 3560 & NA & Normal \\
\hline & 1993 & 7 & 58 & M & NA & $\mathrm{L}$ & 16000 & NA & NA \\
\hline & & & 46 & $\mathrm{~F}$ & Epigastric pain & $\mathrm{L}$ & 14660 & NA & Normal \\
\hline & & & 54 & M & NA & LM & 4587 & NA & NA \\
\hline & & & 72 & $\mathrm{~F}$ & NA & LM & 4110 & NA & NA \\
\hline & & & 87 & M & NA & LM & 2742 & NA & NA \\
\hline & & & 54 & M & NA & $\mathrm{L}$ & 775 & NA & NA \\
\hline & & & 65 & $\mathrm{M}$ & NA & $\mathrm{M}$ & 155 & NA & NA \\
\hline \multirow[t]{2}{*}{ Nagai et al. [28] } & 1993 & 15 & $64.9(52-82)$ & M,10; F,5 & & $\mathrm{L}, 8 ; \mathrm{M}, 6$ & $9 / 9$ & NA & NA \\
\hline & & 13 & $63.0(45-76)$ & M,8; F,5 & & $\begin{array}{c}\mathrm{U}, 1 ; \\
\mathrm{L}, 9 ; \mathrm{M}, 2 \\
\mathrm{U}, 2\end{array}$ & $\begin{array}{l}\text { High } \\
0 / 3\end{array}$ & NA & NA \\
\hline Matsunou et al. [45] & 1994 & 1 & 57 & M & None & $\mathrm{M}$ & 371.7 & NA & 3.2 \\
\hline Aoki et al. [46] & 1994 & 1 & 58 & M & Melena, appetite loss & M & 22000 & NA & NA \\
\hline \multirow[t]{3}{*}{ Aizawa et al. [47] } & 1994 & 3 & 58 & M & NA & $\mathrm{L}$ & 16000 & NA & NA \\
\hline & & & 46 & $\mathrm{~F}$ & NA & $\mathrm{L}$ & 14660 & NA & NA \\
\hline & & & 54 & $\mathrm{M}$ & NA & $\mathrm{L}$ & 4587 & NA & NA \\
\hline Ihling et al. [48] & 1995 & 1 & 64 & NA & Bloated stomach & $\mathrm{L}$ & 79.12 & NA & NA \\
\hline Petrella et al. [49] & 1995 & 1 & 87 & M & Anorexia, BW loss & $\mathrm{L}$ & NA & NA & NA \\
\hline $\begin{array}{l}\text { Morinaga and } \\
\text { Takahashi [50] }\end{array}$ & 1996 & 1 & 62 & M & $\begin{array}{l}\text { BW loss, abdominal } \\
\text { distension }\end{array}$ & $\mathrm{L}$ & 506700 & NA & 5.2 \\
\hline Sugawara et al. [51] & 1996 & 1 & 58 & M & None & $\mathrm{L}$ & 368 & NA & 3.3 \\
\hline Lorenzi et al. [52] & 1996 & 1 & 62 & M & $\begin{array}{c}\text { Fatigue, BW loss, } \\
\text { epigastric pain }\end{array}$ & M & 5596 & NA & 2.5 \\
\hline Ueda et al. [53] & 1996 & 1 & 72 & M & Fatigue & M & 39300 & $>50$ & 3.2 \\
\hline Roberts et al. [54] & 1997 & 1 & 78 & M & Unable to swallow & $\mathrm{L}$ & 2493 & NA & 1.3 \\
\hline \multirow[t]{3}{*}{ Ishikura et al. [55] } & 1997 & 3 & 66 & M & NA & $\mathrm{L}$ & $<5$ & NA & NA \\
\hline & & & 62 & $\mathrm{M}$ & NA & $\mathrm{L}$ & 462000 & NA & 63.3 \\
\hline & & & 69 & M & NA & NA & NA & NA & NA \\
\hline \multirow[t]{2}{*}{ Ejima et al. [56] } & 1997 & 2 & 70 & M & Epigastric pain & $\mathrm{M}$ & 350 & NA & $<1.0$ \\
\hline & & & 65 & M & Fatigue & $\mathrm{M}$ & 4840 & NA & 17.3 \\
\hline Nomura et al. [57] & 1997 & 1 & 66 & $\mathrm{~F}$ & Upper abdominal pain & $\mathrm{M}$ & 2376 & NA & NA \\
\hline Foschini et al. [58] & 1998 & 1 & 80 & $\mathrm{~F}$ & NA & $\mathrm{L}$ & NA & NA & NA \\
\hline Rassidakis et al. [59] & 1998 & 1 & 48 & $\mathrm{M}$ & $\begin{array}{l}\text { Anemia, BW loss, } \\
\text { melena }\end{array}$ & $\mathrm{M}$ & NA & NA & NA \\
\hline
\end{tabular}




\begin{tabular}{|c|c|c|c|c|c|c|c|c|}
\hline \multirow[b]{2}{*}{$\begin{array}{l}\text { CA19-9 } \\
\text { (U/ml) }\end{array}$} & \multirow[b]{2}{*}{$\begin{array}{l}\text { Macroscopic } \\
\text { types }\end{array}$} & \multicolumn{2}{|r|}{ Treatment } & \multirow[b]{2}{*}{$\begin{array}{l}\text { Size } \\
(\mathrm{cm})\end{array}$} & \multirow[b]{2}{*}{ Bile } & \multirow[b]{2}{*}{ Depth $^{3}$} & \multirow[b]{2}{*}{ Metastasis } & \multirow[b]{2}{*}{$\begin{array}{l}\text { Outcome } \\
\text { (months) }\end{array}$} \\
\hline & & Operation & Chemotherapy & & & & & \\
\hline NA & 1 & TG, LR & NA & $8.0 \times 9.0 \times 3.0$ & - & ss & li, ln, lu & \\
\hline NA & 2 & NA & NA & $6.0 \times 6.0$ & - & NA & li, ln, lu, p & \\
\hline NA & 2 & NA & NA & $12 \times 12$ & - & NA & None & 6 Patients died \\
\hline NA & 2 & NA & NA & $5.0 \times 4.5$ & + & NA & li & of the \\
\hline NA & 2 & NA & NA & $7.0 \times 7.0$ & - & NA & li, ln & tumors \\
\hline NA & 3 & NA & NA & $7.0 \times 7.0$ & - & NA & None & \\
\hline NA & 2 & NA & NA & $7.0 \times 5.0$ & - & NA & li, ln, panc & \\
\hline NA & 3 & None & 5-FU, $\mathrm{MMC}^{\mathrm{a}}$ & $8.5 \times 5.5$ & - & se & li, ln, panc & 8, Died \\
\hline NA & Early & NA & NA & $4.0 \times 4.0$ & - & Early & None & NA \\
\hline NA & 3 & NA & NA & $3.0 \times 3.0$ & - & & li & NA \\
\hline 5.2 & 3 & None & $\begin{array}{l}\text { Tegafur, } \\
\text { Adriamycin, MMCa }\end{array}$ & $7.0 \times 7.0$ & + & se & li, ln, lu & 3, Died \\
\hline 17.0 & 3 & TG & & NA & NA & ss & $\ln$ & 6, Died \\
\hline$<7$ & 2 & STG & MMC, UFT ${ }^{\mathrm{a}}$ & $4.0 \times 3.8$ & NA & $\mathrm{mp}$ & $\ln$ & 12, Alive \\
\hline NA & $\overline{1}$ & TG & NA & $3.0 \times 3.0$ & NA & $\mathrm{mp}$ & None & NA \\
\hline NA & NA & None & NA & $14 \times 13 \times 0.8$ & NA & Advanced & li & Died \\
\hline NA & 2 or 3 & GR & None & 9.0 & - & ss & $\ln$ & 16, Died \\
\hline 13 & 1 & TG & MMC, 5-FU, Ara-c ${ }^{\mathrm{a}}$ & $11 \times 7.0 \times 4.0$ & - & ss & None & 120, Alive \\
\hline 470 & 3 & None & $\mathrm{do}^{\mathrm{a}}$ [unknown] & NA & NA & Advanced & & 1.5, Died \\
\hline NA & 2 & PG & NA & 6.0 & - & ss & $\ln$ & 20, Died \\
\hline NA & IIa & PG & $\mathrm{MMC}, \mathrm{ADM}^{\mathrm{b}}$ & $4.3 \times 2.3$ & NA & $\mathrm{sm}$ & $\mathrm{li}, \ln$ & 10, Died \\
\hline NA & IIc & PG & $\begin{array}{l}\text { Cisplatin, MMC, } \\
\text { 5-FU, ADM }{ }^{\mathrm{b}}\end{array}$ & $1.6 \times 1.2$ & NA & $\mathrm{sm}$ & li, $\ln$ & 24, Died \\
\hline NA & IIa & PG, LR & Tegafurc & $4.5 \times 3.3$ & NA & $\mathrm{sm}$ & $\mathrm{li}, \ln$ & 22, Died \\
\hline NA & NA & NA & NA & NA & NA & NA & li & NA \\
\hline NA & IIc & STG & NA & $6.5 \times 4.5$ & NA & $\mathrm{sm}$ & $\ln$ & 17, Alive \\
\hline NA & NA & TG, LR & NA & NA & NA & ss & li, ln, om & 18, Died \\
\hline Normal & 2 & DG & 5-FU, Cisplatin ${ }^{\mathrm{a}}$ & $5.4 \times 4.7$ & & se & Meso colon & 6, Alive \\
\hline NA & 2 & NA & NA & $5.5 \times 4.0$ & - & ss & & 60 , Alive \\
\hline Normal & 2 & STG & UFT $^{d}$ & $4.5 \times 3.7$ & - & $\mathrm{mp}$ & li, ln, lu & 14, Died \\
\hline NA & 2 & NA & NA & $7.5 \times 6.5$ & - & se & $\ln$ & 12, Alive \\
\hline NA & 2 & NA & NA & $6.5 \times 4.0$ & - & ss & li, $\ln$ & 18, Died \\
\hline NA & 2 & NA & NA & $6.5 \times 6.2$ & - & ss & $\mathrm{li}, \ln$ & 20, Died \\
\hline NA & 2 & NA & NA & $5.0 \times 0.5$ & - & ss & li & 12, Died \\
\hline NA & 2 & NA & NA & $5.0 \times 4.5$ & - & ss & li, $\ln$ & 40, Died \\
\hline NA & & NA & $\begin{array}{l}\text { NA } \\
\text { none, } 3\end{array}$ & $5.2(2-10)$ & & $\begin{array}{l}\text { Early } 5 ; \\
\quad \text { advanced } 10\end{array}$ & $\begin{array}{l}\ln , 12 \\
\text { li,3; }\end{array}$ & \\
\hline NA & & NA & & $7.5[2.5-13]$ & & $\begin{array}{l}\text { Early 0; } \\
\quad \text { advanced } 13\end{array}$ & $\begin{array}{l}\ln , 8 ; \\
\text { li,5; none } 3\end{array}$ & \\
\hline NA & $\mathrm{IIa}+\mathrm{c}$ & PG & None & $3.2 \times 2.5$ & NA & Early & None & 39, Alive \\
\hline NA & 1 & TG, LR & $\begin{array}{l}\text { MAC-VD } \\
\text { ADM, lipiodol }\end{array}$ & $7.0 \times 10 \times 2.2$ & NA & se & li, ln & 3, Died \\
\hline NA & NA & NA & NA & $5.5 \times 4.0$ & NA & NA & $\ln$ & 96, Alive \\
\hline NA & NA & NA & NA & $4.5 \times 3.7$ & NA & NA & $\mathrm{li}, \ln$ & 7, Died \\
\hline NA & NA & NA & NA & $7.5 \times 6.5$ & NA & NA & $\ln$ & 8, Alive \\
\hline NA & 2 or 3 & DG & $\begin{array}{l}\text { 5-FU, farmorubicin, } \\
\text { leucovorin }^{\mathrm{a}}+\text { rad1 }\end{array}$ & 10 & NA & se & $\ln$, om & 12, Died \\
\hline NA & Polypoid & STG & NA & 5.0 & - & se & None & 6, Died \\
\hline NA & 2 & None & None & $7.2 \times 4.5$ & - & $\mathrm{mp}$ & li, ln, om & 1.3, Died \\
\hline NA & $\mathrm{IIa}+\mathrm{c}$ & STG & None & $2.8 \times 1.7$ & + & $\mathrm{sm}$ & $\ln$ & 12, Alive \\
\hline 10 & NA & STG & 5-FU, ADM, MMC & 5.0 & NA & se & $\mathrm{li}, \ln$ & 26, Died \\
\hline NA & 1 & None & Epirubicin, lipiodol ${ }^{\mathrm{b}}$ & $12 \times 10 \times 8.0$ & + & se & li, $\ln$ & 5, Died \\
\hline 7.0 & NA & STG & NA & $4.0 \times 3.0 \times 2.0$ & + & se & $\ln$ & 12, Alive \\
\hline NA & 3 & TG, LR & None & NA & NA & ss & li, ln & 13 , Alive \\
\hline NA & 1 & None & NA & NA & - & ss & li, lu & 1, Died \\
\hline NA & NA & None & NA & NA & NA & NA & $\mathrm{li}$ & Died \\
\hline 9.5 & 1 & TG & NA & $8.0 \times 7.0 \times 3.0$ & - & se & li, ln & 3, Died \\
\hline 444.5 & 3 & None & NA & $3.0 \times 3.0 \times 2.5$ & - & $\mathrm{se}$ & li, ln, lu, p & 3, Died \\
\hline NA & 3 & PG & 5-FU, CDDPa & $10 \times 10$ & NA & ss & $\ln$ & 3, Alive \\
\hline NA & Polypoid & NA & $\mathrm{NA}$ & 10.0 & - & NA & NA & NA \\
\hline NA & 3 & TG & $\begin{array}{l}\text { Doxorubicin, MMC, } \\
5-\mathrm{Fu}^{\mathrm{a}}+\mathrm{rad}^{2}\end{array}$ & $7.0 \times 5.5$ & NA & Whole layer & $\ln$ & 12, Alive \\
\hline
\end{tabular}


Table 1. continued

\begin{tabular}{|c|c|c|c|c|c|c|c|c|c|}
\hline \multirow[b]{2}{*}{ Author } & \multirow[b]{2}{*}{ Year } & \multirow[b]{2}{*}{$\begin{array}{l}\text { Number of } \\
\text { patients }\end{array}$} & \multirow[b]{2}{*}{$\begin{array}{c}\text { Age } \\
\text { (years) }\end{array}$} & \multirow[b]{2}{*}{ Sex } & \multirow[b]{2}{*}{ Chief complaints } & \multirow[b]{2}{*}{ Location } & \multicolumn{3}{|c|}{ Tumor marker } \\
\hline & & & & & & & $\begin{array}{c}\mathrm{AFP} \\
(\mathrm{ng} / \mathrm{ml})\end{array}$ & $\begin{array}{l}\text { PIVKA-II } \\
(\mathrm{U} / \mathrm{ml})\end{array}$ & $\begin{array}{c}\text { CEA } \\
(\mathrm{ng} / \mathrm{ml})\end{array}$ \\
\hline Nemoto et al. [60] & 1998 & 1 & 62 & M & Epigastric discomfort & M & 9554 & NA & 117 \\
\hline Kang and Kim [61] & 1998 & 1 & 55 & $\mathrm{~F}$ & Epigastric discomfort & M & 630 & NA & NA \\
\hline Suzuki et al. [62] & 1999 & 1 & 73 & $\mathrm{M}$ & Epigastric pain & ML & 296.8 & NA & 3.2 \\
\hline Present patient & & 1 & 67 & M & Fatigue & LM & 10084 & NA & 6 \\
\hline
\end{tabular}

Macroscopic type and the depth are according to the Japanese classification of gastric carcinoma [25]

NA, details not available; BW, body weight; U, upper-third portion; M, middle-third portion; L, lower-third portion; $\mathrm{U}^{1}$, remnant stomach; TG, total gastrectomy; STG, subtotal gastrectomy; DG, distal gastrectomy; PG, partial gastrectomy; GR, gastric resection; LR, liver resection; li, liver; ln, lymph nodes; lu, lung; p, peritoneum; panc, pancreas; om, omentum; 5-FU, 5-fluorouracil; MMC, mitomycin C; ADM, adriamycin; AFP, alpha-fetoprotein; PIVKA-II, des- $\gamma$-carboxy prothrombin; CEA, carcinoembryonic antigen; CA 19-9, carbohydrate artigen 19-9; TS-1, 1M tegafur-0.4 M gimestat-1 M ostat potassium; CDDP, cisplatin; 5'DFUR, 5'-deoxy-5-fluorouridine; UFT, tegafur uracil; rad ${ }^{1}$, intraoperative radiation therapy; $\mathrm{rad}^{2}$, postoperative radiation therapy; died, died of the disease

A
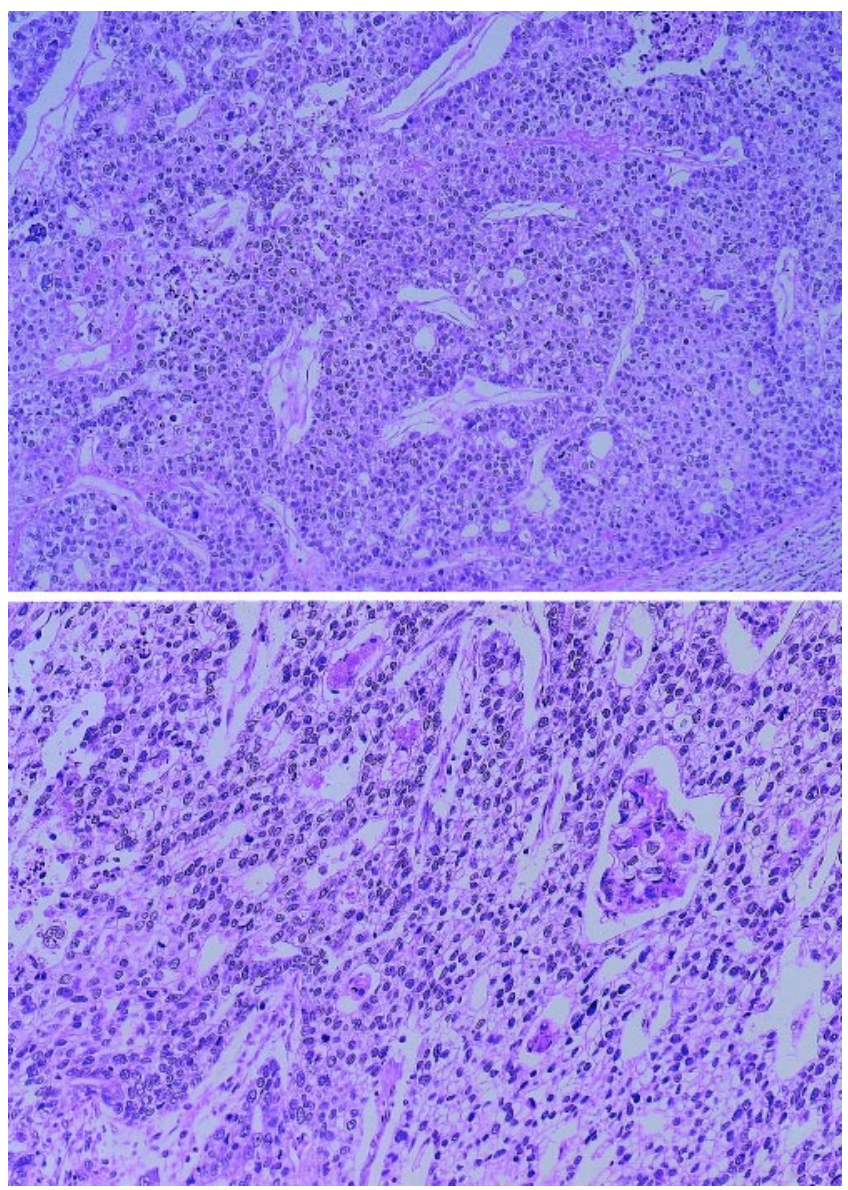

Fig. 4. A The major part of the tumor was composed of cords of polygonal cells with voluminous nuclei and prominent nucleoli, and resembled moderately differentiated hepatocellular carcinoma; B the minor part of the tumor, showing fetal-type adenocarcinoma with clear cell cytoplasm. A and $\mathbf{B}$ $\mathrm{H} \& \mathrm{E}, \times 100$

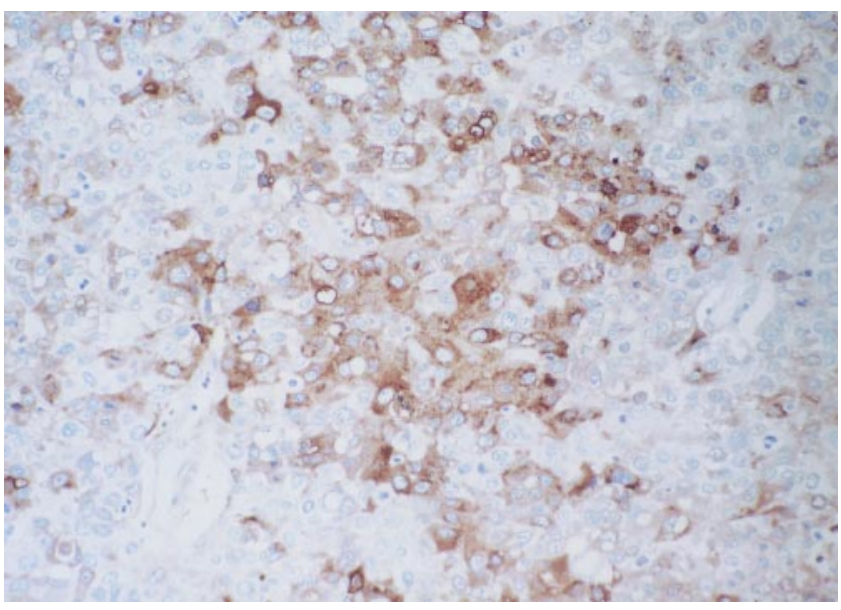

Fig. 5. Immunohistochemical staining for alpha-fetoprotein (AFP) shows diffuse positivity in the hepatoid areas. $\times 200$

increased again to $22.5 \mathrm{ng} / \mathrm{ml}$. Careful follow-up of this patient has revealed growth of the liver metastases, but the patient remains alive 11 months after the operation.

\section{Discussion}

AFP is an oncofetal glycoprotein produced mainly in the fetal liver and the yolk sac and, to a small extent, in the fetal gastrointestinal tract [1]. The highest serum levels occur between weeks 12 and 15 of gestation. After birth, the protein level in the serum rapidly decreases. However, under pathologic conditions, AFP is detected in the serum of adults with hepatocellular carcinoma, those with yolk sac tumors, and those with noncancerous liver disease associated with liver regeneration, such as cirrhosis and hepatitis [2-6]. AFPProducing malignant tumors have been reported in several organs; for example, the lung $[7,8]$, pancreas $[9,10]$, 


\begin{tabular}{|c|c|c|c|c|c|c|c|c|}
\hline \multirow[b]{2}{*}{$\begin{array}{l}\text { CA19-9 } \\
\text { (U/ml) }\end{array}$} & \multirow[b]{2}{*}{$\begin{array}{l}\text { Macroscopic } \\
\text { types }\end{array}$} & \multicolumn{2}{|r|}{ Treatment } & \multirow[b]{2}{*}{$\begin{array}{l}\text { Size } \\
(\mathrm{cm})\end{array}$} & \multirow[b]{2}{*}{ Bile } & \multirow[b]{2}{*}{ Depth $^{3}$} & \multirow[b]{2}{*}{ Metastasis } & \multirow[b]{2}{*}{$\begin{array}{l}\text { Outcome } \\
\text { (months) }\end{array}$} \\
\hline & & Operation & Chemotherapy & & & & & \\
\hline NA & 3 & DG & $\begin{array}{l}\text { 5-FU, CDDPa } \\
\quad+\text { epirubicin, } \mathrm{MMC}^{\mathrm{b}}\end{array}$ & NA & NA & se & li, ln & 25, Died \\
\hline NA & NA & TG & None & $4.0 \times 4.0$ & - & ss & $\ln$ & 7, Alive \\
\hline NA & 5 & DG & $5-\mathrm{FU}^{\mathrm{b}}$ & $8.0 \times 8.0$ & - & $\mathrm{se}$ & li, $\ln$ & 50, Alive \\
\hline 4 & 5 & TG & TS-1 & $8.5 \times 8.5$ & - & ss & li, $\ln$ & 8, Alive \\
\hline
\end{tabular}

a Systemic chemotherapy

${ }^{\mathrm{b}}$ Intra-arterial chemotherapy

c Oral chemotherapy

${ }^{\mathrm{d}}$ Various kinds of anticancer drug were given, i.e., MAC-VD,

$\mathrm{MMC}+\mathrm{ADM}+\mathrm{CDDP}+5^{\prime} \mathrm{DFUR}$

colon [11], bladder [12], and ovary [13]. The stomach is one of the most common sites affected by these tumors, and the first such case was described by Bourreille et al. in 1970 [15]. The reported incidence of AFP-producing gastric carcinoma has been $1.3 \%-15 \%$ of all gastric carcinomas [16-20].

Kodama et al. [21] described two histologic types of AFP-producing gastric carcinoma, based on immunohistochemical staining; a medullary type, characterized by polygonal cells arranged in solid nests or sheets, with scattered large pleomorphic or multinucleated giant cells, and a well differentiated papillary or tubular type with clear cytoplasm. The two types sometimes coexisted in a single tumor. Ishikura et al. [22,23] proposed the term "hepatoid adenocarcinoma of the stomach" for primary gastric carcinomas characterized histologically by hepatoid differentiation and the production of large amounts of AFP. Motoyama et al. [26] reported that hepatoid adenocarcinoma was the most frequently observed histological type of AFP-producing gastric cancer. To discriminate between hepatoid adenocarcinoma and nonhepatoid adenocarcinoma of the stomach, the measurement of AFP isoforms is also useful, and the AFP level in the serum of patients with hepatoid adenocarcinona shows a liver-type binding pattern with the lectin concanavalin A (Con-A) [23]. However, Ooi et al. [27] reported that this was not significant, because such a liver-type binding pattern was detected in only $20 \%$ of AFP-producing tumors.

On the other hand, Nagai et al. [28] noted that the diagnosis of hepatoid adenocarcinoma of the stomach was not dependent on whether AFP was produced, and considered that diagnosis was better based on the recognition of the characteristic histologic features. They reported that the presence of PAS-positive and diastase-resistant intracytoplasmic eosinophilic globules, and immunohistochemical positivity for AAT,
ACT, and bile (which is shown in hepatocellular carcinoma) supported the diagnosis, even though these findings were nonspecific.

Furthermore, there is sometimes confusion about whether hepatoid carcinoma originates from the stomach or the liver, because most patients show multiple liver metastasis preoperatively. Our patient's tumor was believed to have originated from hepatoid adenocarcinoma of the stomach, because his serum AFP level decreased rapidly after gastrectomy (although this level has again been increasing) and the liver tumor was not resected. This rapid decrease was probably due to mass reduction by surgery, while the postoperative chemotherapy with TS-1 may be relatively effective for the suppression of tumor growth. Furthermore, although the size of the liver tumors has increased, the patient's serum CEA level was not increased just before and after surgery. Additionally, most primary liver cancers in Japan have a background of cirrhosis or fibrosis in the noncancerous portion of the liver [29]. However, our patient was negative for hepatitis B surface antigen and antibody, and for hepatitis $\mathrm{C}$ antibody, and no cirrhosis or fibrosis was seen in the noncancerous part of the liver.

In the literature, 85 cases described by the term "hepatoid adenocarcinoma of the stomach" have been reported, including the present case (Table 1 [22,23,24, $26,28,30-62])$. The average age of the patients was 63.5 years (range, $44-87$ years), and the male-to-female ratio was 58:25 (the sex was not stated for 2 patients). In most of these patients, the tumors occurred mainly at the antrum $(60.2 \%)$. The average serum AFP level was $51130.1 \mathrm{ng} / \mathrm{ml}$ (range, less than 1.0-700000 ng/ml); that is, much higher than normal. Although there were no major symptoms sufficient to allow diagnosis of this type of cancer, epigastric pain and general fatigue, because of anemia, were the most common symptoms. The average maximal tumor diameter was $6.5 \mathrm{~cm}$ (range, 1.6-14.0 cm). Early-stage cancer was seen in 
only 11 patients; the others were advanced stage. Although type 1 and type 5 tumors were reported, the tumors in about two-thirds of the 47 patients with clearly described gross findings (29 patients; 61.7\%) were classified as type 2 or 3 . In addition, coagulation necrosis was frequently observed. In most of the reported patients, metastases to the liver and/or lymph nodes were detected preoperatively. Bile secretion was reported in only 4 patients, whereas this is a common feature in hepatocellular carcinoma. Furthermore, most of the patients died within 2 years of surgery, despite systemic chemotherapy having been administered in several patients. These reports suggest that even earlystage hepatoid adenocarcinoma has an extremely poor prognosis, because of the frequent occurrence of liver and/or lymph node metastases [24,26,28].

However, the reasons for the poor prognosis are not clearly understood. Nagai et al. [28] reported that hepatoid adenocarcinoma had a poor prognosis compared with that for AFP-producing nonhepatoid adenocarcinoma. One possibility is that hepatoid adenocarcinoma produces AAT and/or ACT as well as AFP. AAT and ACT have immunosuppressive and protease-inhibitory properties that enhance invasiveness $[63,64]$. Also, AFP has a suppressive effect on lymphocyte transformation [65]. In addition, Koide et al. [66] have reported that AFP-producing gastric cancer has high proliferative activity, weak apoptosis, and rich neovascularization. Compared with findings in previous reports [66,67], Ki-67 expression-which is associated with proliferative activity and tumor progression [68] was extremely marked in our patient. Furthermore, these tumors are known to be resistant to chemotherapy [69].

Because of the extremely poor prognosis of hepatoid adenocarcinoma of the stomach, it is important to ensure that the diagnosis is accurate. Furthermore, as Chang et al. [24] have reported, even if no metastasis is present preoperatively, liver metastasis can occur within a year after surgery, and, thus, close observation and long-term follow-up of patients are required. However, several patients for whom chemotherapy was effective have been reported $[34,38,60,62]$. Therefore, even if hepatoid adenocarcinoma is diagnosed, curative resection and further chemotherapy are recommended.

\section{References}

1. Gitlin D, Pericelli A, Gitlin GM. Synthesis of alpha-fetoprotein by liver, yolk sac, and gastrointestinal tract of the human conceptus. Cancer Res 1972;32:979-82.

2. Abelev GI. Production of embryonal serum alpha-globulin by hepatomas: review of experimental and clinical data. Cancer Res 1968;28:1344-50.
3. Purves LR, Bersohn I, Geddes EW. Serum alpha-fetoprotein and primary cancer of the liver in man. Cancer 1970;25:1261-70.

4. Breborowicz J, Mackiewicz A, Breborowicz D. Microheterogeneity of alpha-fetoprotein in patient serum as demonstrated by lectin affinoelectrophoresis. Scand J Immunol 1981;14:15-20.

5. Ezaki T, Yukaya H, Ogawa Y, Chang YC, Nagasue N. Evaluation of alpha-fetoprotein level without evidence of recurrence after hepatectomy for hepatocellular carcinoma. Cancer 1988;61: 1880-3.

6. Ganjei P, Nadji M, Albores-Saavedra J, Morales AR. Histologic markers in primary and metastatic tumors of the liver. Cancer 1988:62:1994-8.

7. Asamura H, Nakayama H, Kondo H, Tsuchiya R, Ono R, Noguchi M, et al. AFP-producing squamous cell carcinoma of the lung in an adolescent. Jpn J Clin Oncol 1996;26:103-6.

8. Okunaka T, Kato H, Konaka C, Yamamoto H, Furukawa K. Primary lung cancer producing alpha-fetoprotein. Ann Thorac Surg 1992;53:151-2.

9. Congy F, Pelletier S, Langlois P, Khayat D, Homsi T, Emerit J. Pancreatic endocrine tumor with metastases and increase of alpha-fetoprotein: a case report. Rev Med Interne 1992;13:4513.

10. Itoh T, Kishi K, Tojo M, Kitamura N, Kinoshita Y, Inatome T, et al. Acinar cell carcinoma of the pancreas with elevated serum alpha-fetoprotein levels: a case report and a review of 28 cases reported in Japan. Gastroenterol Jpn 1992;27:785-91.

11. Kato K, Masuda M, Ingu A, Imai M, Kasai S, Mito M, et al. Colon cancer with a high serum alpha-fetoprotein level. Am J Gastroenterol 1996;91:1045-6.

12. Yamada K, Fujioka Y, Ebihara Y, Kiriyama I, Suzuki H, Akimoto M. Alpha-fetoprotein-producing undifferentiated carcinoma of the bladder. J Urol 1994;152:958-60.

13. Hammad A, Jasnosz KM, Olson PR. Expression of alphafetoprotein by ovarian Sertoli-Leydig cell tumors: case report and review of the literature. Arch Pathol Lab Med 1995;119:1075-9.

14. McIntire KR, Waldmann TA, Moertel CG, Go VLW. Serum $\alpha$ fetoprotein in patients with neoplasms of gastrointestinal tract. Cancer Res 1975;35:991-6.

15. Bourreille J, Metayer P, Sauger F, Fondimare A. Existence d'alpha fetoproteine au cours d'un cancer secondaire du foie d'origine gastrique. Als Press Med 1970;78:1277-8.

16. McIntire KR, Waldmann TA, Moertel CG, Go VLW. Serum alpha-fetoprotein in patients with neoplastic carcinomas. N Engl J Med 1971;285:1060-1.

17. Akai S, Kato K. Serum $\alpha$-fetoprotein-positive stomach cancer. Gann Monogr 1973;14:149-54.

18. Takahashi Y, Mai O, Ogino T, Ueda H, Sawaguchi K, Ueno M. Clinicopathological study of AFP-producing gastric cancer. Significance of AFP in gastric cancer. J Jpn Surg Soc 1987;88:696700 .

19. Yonemura $\mathrm{Y}$, Hashimoto $\mathrm{T}$, Sawa $\mathrm{T}$, Shima $\mathrm{Y}$, Kamata $\mathrm{T}$, Nishimura $G$, et al. The significance of measurement of serum CEA, AFP and hCG in gastric cancer patients (in Japanese with English abstract). J Jpn Soc Clin Surg 1987;48:174-9.

20. Nishio Y, Urakawa T, Nakamoto M, Yamaguchi T, Tanaka H, Idei $\mathrm{H}$, et al. Study of nine cases of $\alpha$-fetoprotein (AFP) producing gastric cancer (in Japanese with English abstract). J Jpn Soc Clin Surg 1989;50:1176-80.

21. Kodama T, Kameya T, Hirota T, Shimosato Y, Ohkura H, Mukojima $\mathrm{T}$, et al. Production of $\alpha$-fetoprotein, normal serum proteins, and human chorionic gonadotropin in stomach cancer: histologic and immunohistochemical analysis of 35 cases. Cancer 1981:4:1647-55.

22. Ishikura H, Fukasawa Y, Ogasawara K, Natori T, Tsukada Y, Aizawa M. An AFP-producing gastric carcinoma with features of hepatic differentiation: a case report. Cancer 1985;56:840-8.

23. Ishikura H, Kirimoto K, Shamoto M, Miyamoto Y, Yamagiwa $H$, Itho $\mathrm{T}$, et al. Hepatoid adenocarcinomas of the stomach: an analysis of seven cases. Cancer 1986;58:119-26. 
24. Chang YC, Nagasue N, Abe S, Kohno H, Kumar DD, Nakamura T. $\alpha$-Fetoprotein-producing early gastric cancer with liver metastasis: report of three cases. Gut 1991;32:542-5.

25. Japanese Gastric Cancer Association. Japanese classification of gastric carcinoma (in Japanese). 13th ed. Tokyo: Kanehara;1999.

26. Motoyama T, Aizawa K, Watanabe H, Fukase M, Saito K. $\alpha$ Fetoprotein-producing gastric carcinomas: a comparative study of three different subtypes. Acta Pathol Jpn 1993;43:654-61.

27. Ooi A, Nakanishi I, Sakamoto N, Tsukada Y, Takahashi Y, Minamoto T, et al. Alpha-fetoprotein (AFP)-producing gastric carcinoma. Is it hepatoid differentiation? Cancer 1990;65:1741-7.

28. Nagai E, Ueyama T, Yao T, Tsuneyoshi M. Hepatoid adenocarcinoma of the stomach. Cancer 1993;72:1827-35.

29. Okuda K and the Liver Cancer Study Group of Japan. Primary liver cancers in Japan. Cancer 1980;45:2663-9.

30. Ebihara T, Koyama S, Hirai S, Nakahara A, Tanaka N, Yamagata $\mathrm{Y}$, et al. An autopsy case of AFP-producing gastric carcinoma resembling hepatocellular carcinoma on light microscopy (in Japanese). Gan No Rinsho (Jpn J Cancer Clin) 1987;33:399-405.

31. Ishikura H, Mizuno K, Shamoto M, Kirimoto K, Tsukada H, Ito $\mathrm{T}$, et al. Hepatoid adenocarcinoma of the stomach-the nature of the disease; its entity and its clinicopathology (in Japanese with English abstract). Stomach and Intestine 1987;22:75-83.

32. Yamada H, Nakanishi T, Kishino B, Sakata Y. A case report of so-called gastric hepatoid adenocarcinoma with liver metastasis (in Japanese). Sumitomoishi 1987;14:94-9.

33. Watanabe M, Takano K, Hamada Y, Matsushima T, Miyazaki T, Katoh $\mathrm{H}$, et al. A case of AFP-producing gastric cancer accompanied with testicular tumor (in Japanese). Gan No Rinsho (Jpn J Cancer Clin) 1988;34:2131-7.

34. Ohashi N, Goshima H, Yamazaki H, Ota M, Kusakawa M, Soga T. A case report of so-called hepatoid adenocarcinoma of the stomach without liver metastasis (in Japanese). Ringe (J Clin Surg) 1988;43:1537-41.

35. Yamagiwa H. "From the surgical specimens." XGV: a case of gastric remnant carcinoma (hepatoid carcinoma) (in Japanese). Mie Igaku 1988;32:357-8.

36. Li XZ. A case of hepatoid adenocarcinoma of the stomach (in Chinese). Chung Hua Chung Liu Tsa Xhih 1989;11:74-5.

37. Matias-Guiu X, Guix M. Hepatoid gastric adenocarcinoma. Pathol Res Pract 1989;185:397-400.

38. Takeuchi M, Yamaoka S, Matsumura K, Kohara T, Nishigi S, Maekawa M. An alpha-fetoprotein-producing gastric carcinoma with hepatoid features. A case report of "hepatoid adenocarcinoma of the stomach" (in Japanese with English abstract). Gastroenterol Endosc 1989;31:442-8.

39. Miyake S, Ebihara Y, Serizawa H, Takeuchi K, Yamada K, Miyata $\mathrm{H}$, et al. Gastric carcinoma with a raised serum AFP $(\alpha-$ fetoprotein), with special reference to its exofoliative cytology (in Japanese with English abstract). J Jpn Soc Clin Cytol 1989;28:70-4.

40. Caruso RA. Hepatoid gastric adenocarcinoma. A histological and immunohistochemical study of a case. Eur J Appl Histochem 1991;35:203-9.

41. Votte A, Sevestre H, Dupas JL, Joly JP, Bental A, Delamarre J, Capron JP. Hepatoid adenocarcinoma of the stomach. Gastroenterol Clin Biol 1991;15:437-40.

42. Kudo M, Takamine Y, Nakamura K, Shirane H, Uchida H, Kasakura S, et al. Des- $\gamma$-carboxy prothrombin (PIVKA-II) and $\alpha$-fetoprotein-producing IIc-type early gastric cancer. Am J Gastroenterol 1992;87:1859-62.

43. de Lorimier A, Park F, Aranha GV, Reyes C. Hepatoid carcinoma of the stomach. Cancer 1993;71:293-6.

44. Kanda T, Sasaki J, Nashimoto A, Suzuki M, Tsunoda H, Muto T. A giant tumor of the mesocolon found to be metastasis from alpha-fetoprotein-producing gastric carcinoma: report of a case. Surg Today 1993;23:1006-9.

45. Matsunou H, Konishi F, Jalal REA, Yamauchi N, Mukawa A. Alpha-fetoprotein-producing gastric carcinoma with enteroblastic differentiation. Cancer 1994;73:534-40.
46. Aoki S, Kuno Y, Adachi T, Adachi N, Miyoshi T, Shimokawa K, et al. A case of alpha-fetoprotein-producing hepatoid gastric cancer. Geka (Surgery) 1994;56:989-92.

47. Aizawa K, Motoyama T, Suzuki S, Tanaka N, Yabusaki H, Tanaka S, et al. Different characteristics of hepatoid and nonhepatoid $\alpha$-fetoprotein-producing gastric carcinomas: an experimental study using xenografted tumors. Int J Cancer 1994;58: 430-5.

48. Ihling C, Schaefer HE, Baumgartner U, Riede UN. Hepatoid adenocarcinoma of the stomach: a case report. Gen Diagn Pathol 1995;141:61-5.

49. Petrella $T$, Montagnon $J$, Roignot $P$, van Nieuvenhuyse A, Matagrin C, Michels-Marzais D, et al. Alpha-fetoproteinproducing gastric adenocarcinoma. Histopathology 1995;26:171-5.

50. Morinaga S, Takahashi Y. Primary hepatocellular carcinoma and hepatoid adenocarcinoma of the stomach with liver metastasis: an usual association. Jpn J Clin Oncol 1996;26:258-63.

51. Sugawara Y, Konishi T, Hiraishi M, Sato A, Natomi H, Aoki T, et al. Hepatoid adenocarcinoma of the stomach: a case report. Hepatogastroenterology 1996;43:995-9.

52. Lorenzi M, Vindigini C, Tripodi SA, Megha T, Mancini S, Francini G. Hepatoid gastric carcinoma. A case report. Int J Biol Markers 1996;11:216-9.

53. Ueda H, Yoshikawa N, Hashimoto T, Yamaguchi T, Dousei T, Moriguchi A, et al. A case of liver metastasis from so-called hepatoid adenocarcinoma of stomach with lipiodol accumulation. Jpn J Gastroenterol 1996;93:120-5.

54. Roberts CC, Colby TV, Batts KP. Carcinoma of the stomach with hepatocyte differentiation (hepatoid adenocarcinoma). Mayo Clin Proc 1997;72:1154-60.

55. Ishikura H, Kishimoto T, Andachi H, Kakuta Y, Yoshiki T. Gastrointestinal hepatoid adenocarcinoma: venous permeation and mimicry of hepatocellular carcinoma, a report of four cases. Histopathology 1997;31:47-54.

56. Ejima S, Mizukami Y, Miyamoto K, Sogawa K, Sugita A, Ueda N. Three cases of AFP-producing gastric cancer-immunohistochemical study (in Japanese with English abstract). Ehime Igaku 1997;16:38-48.

57. Nomura H, Aragai J, Tamai O, Isa T, Shiraishi M, Kusano T, et al. Alpha-fetoprotein-producing gastric cancer: a case report and a brief literature review. Ryukyu Med J 1997;17:22932 .

58. Foschini MP, Baccarini P, Monte PRD, Sinard J, Eusebi V, Rosai J. Albumin gene expression in adenocarcinomas with hepatoid differentiation. Virchows Arch 1998;433:537-41.

59. Rassidakis GZ, Delladetsima JK, Letsos SP, Polyzos A, Yannopoulos A. Hepatoid adenocarcinoma of the stomach with extensive neuroendocrine differentiation and a coexisting carcinoid tumor. Histopathology 1998;33:186-8.

60. Nemoto A, Goshima H, Tanigawa K, Hayashi J, Ikeda G. A case of AFP-producing gastric cancer with multiple liver metastasis showing marked improvement by hepatoarterial infusion with cisplatin (in Japanese). Mie Igaku 1998;42:84-94.

61. Kang GH, Kim YI. $\alpha$-Fetoprotein-producing gastric carcinoma presenting focal hepatoid differentiation in metastatic lymph nodes. Virchows Arch 1998;432:85-7.

62. Suzuki Y, Watanabe M, Nonaka H, Kase H, Tokura N, Kikuchi M, et al. A case of advanced gastric carcinoma producing alpha-fetoprotein with multiple liver metastasis in which intrahepatic chemotheraphy was effective postgastrectomy (in Japanese with English abstract). Jpn J Gastroenterol Surg 1999;32:23659.

63. Pasternack MS, Eisen HN. A novel serum esterase expressed by cytotoxic T lymphocytes. Nature 1985;314:743-5.

64. Redelman D, Hudig D. The mechanism ot cell-mediated cytotoxicity. I. Killing by murine cytotoxic T lymphocytes requires cell surface thiols and activate proteases. J Immunol 1980;124:870-8.

65. Yachnin S. The immunosuppressive properties of alpha-fetoprotein: a brief overview. Ann NY Acad Sci 1983;417:105-7. 
66. Koide N, Nishio A, Igarashi J, Kajikawa S, Adachi W, Amano J. $\alpha$-Fetoprotein-producing gastric cancer: histochemical analysis of cell proliferation, apoptosis, and angiogenesis. Am J Gastroenterol 1999;94:1658-63.

67. Kakeji Y, Korenaga D, Tsujitani S, Haraguchi M, Maehara Y, Sugimachi K. Predictive value of Ki-67 and argyrophilic nucleolar organizer region staining for lymph node metastasis in gastric cancer. Cancer Res 1991;51:3503-6.
68. Gerdes J, Lemke H, Baisch H, Wacker HH, Schwab U, Stein H. Cell cycle analysis of a cell proliferation-associated human nuclear antigen defined by monoclonal antibody Ki-67. J Immunol 1984;133:1710-5.

69. Chang YC, Nagasue N, Kohono H, Ohiwa K, Yamanoi A, Nakamura T. Xenotransplantation of alpha-fetoprotein-producing gastric cancers into nude mice: characteristics and responses to chemotherapy. Cancer 1992;69:872-8. 